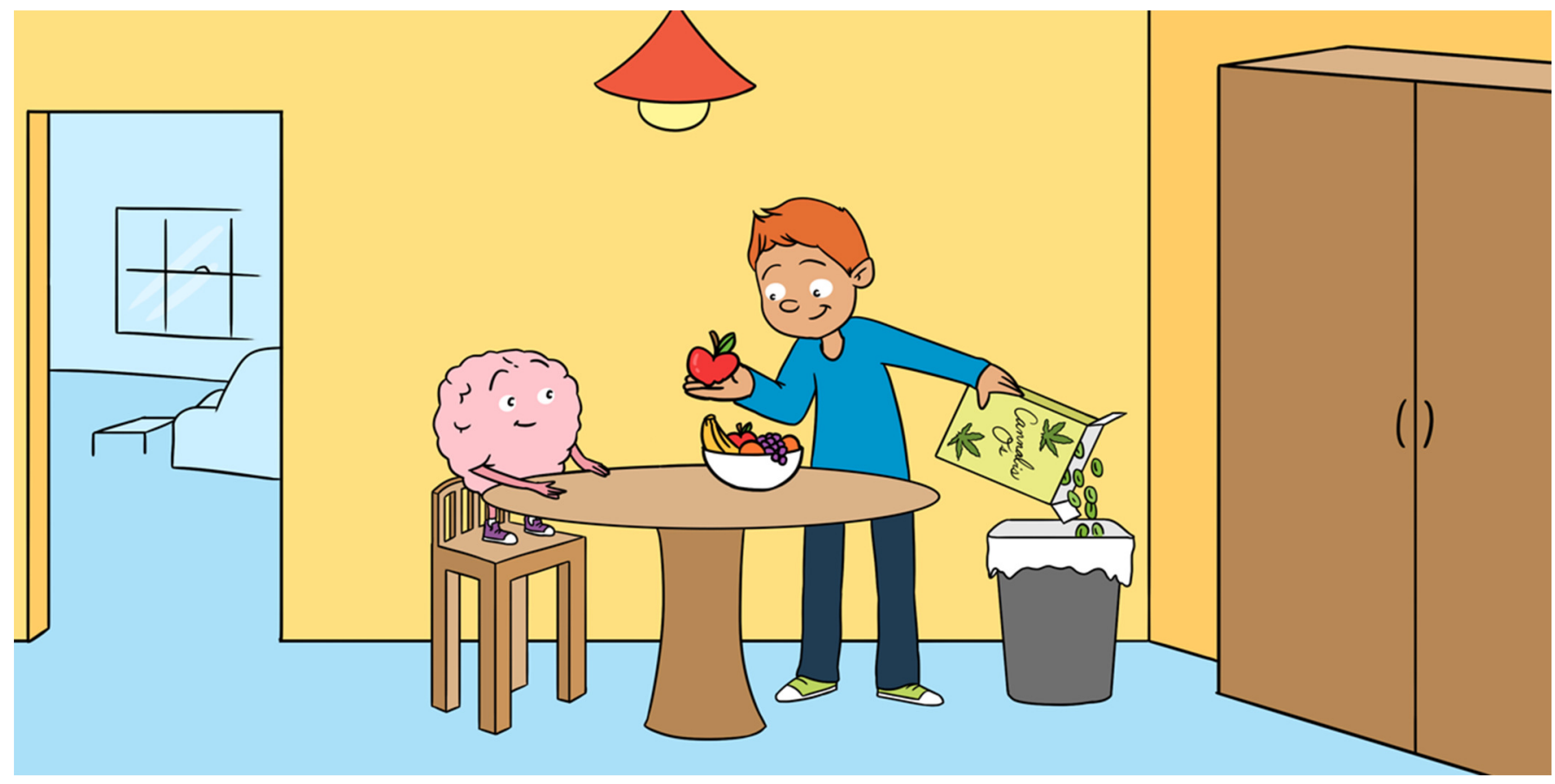

\title{
BE CAREFUL WHAT YOU FEED YOUR BRAIN: CANNABIS AND MENTAL HEALTH
}

\section{Srivaishnavi Loganathan ${ }^{1,2^{*} t}$, Dorothee Pöhlchen ${ }^{1,3^{*} t}$, Elena Brivio ${ }^{1,2 \dagger}$, Ashley L. Comes ${ }^{1,4}$, Shalaila S. Haas ${ }^{1,5}$, Janos L. Kalman ${ }^{1,4,6}$, Anthi C. Krontira ${ }^{1,3}$, Fabian Stamp ${ }^{1,2,3}$, Eva Hoch ${ }^{6,7}$ and Carsten T. Wotjak ${ }^{2,8 \neq}$}

\footnotetext{
1 International Max Planck Research School for Translational Psychiatry, Max Planck Institute of Psychiatry, Munich, Germany ${ }^{2}$ Department of Stress Neurobiology and Neurogenetics, Max Planck Institute of Psychiatry, Munich, Germany

${ }^{3}$ Department of Translational Research in Psychiatry, Max Planck Institute of Psychiatry, Munich, Germany

${ }^{4}$ Institute of Psychiatric Phenomics and Genomics, University Hospital, LMU Munich, Munich, Germany

${ }^{5}$ Department of Psychiatry, Icahn School of Medicine at Mount Sinai, New York, NY, United States

${ }^{6}$ Department of Psychiatry and Psychotherapy, University Hospital, LMU Munich, Munich, Germany

${ }^{7}$ Division of Clinical Psychology and Psychological Treatment, Department of Psychology, LMU Munich, Munich, Germany

${ }^{8}$ Research Group "Neuronal Plasticity", Max-Planck Institute of Psychiatry, Munich, Germany
}

\section{YOUNG REVIEWERS:}

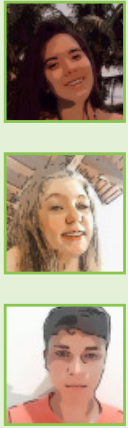

ANA CLARA

AGE: 14

ANA LUISA

AGE: 15

HENRIQUE

AGE: 15
Adolescence is an important period when the brain undergoes many changes. Because the brain is changing so much at this time, our experiences can have a huge impact on the brain's health. Positive experiences keep the mind healthy, whereas negative experiences can lead to mental disorders. To process all the information that comes into the brain, the brain cells must talk with each other. They communicate via messenger systems. One of these systems is called the endocannabinoid ( $\mathrm{eCB}$ ) system. The $\mathrm{eCB}$ system plays an important role in the changes that happen in the adolescent brain. In this article, we see how the $\mathrm{eCB}$ system is affected by cannabis and how this may lead to a mental disorder. 


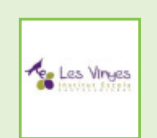

INSTITUT-

ESCOLA

LES VINYES

AGE: 15

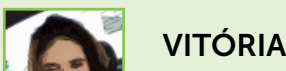

AGE: 14
PRUNING

Removal of connections between neurons that are weak or not in use.

\section{BEING MINDFUL ABOUT BODY AND MIND: CAN THE MIND BE ILL?}

Imagine a healthy person. You are probably thinking of an energetic person who is able to run, play, eat, and sleep well. Now imagine an unhealthy person. You probably picture someone with a snotty nose or a broken arm. But what about people who cannot go grocery shopping because they are extremely anxious? Or people who cannot find the energy to get out of bed? Although their illnesses are not visible to the eye, they are also suffering. Illnesses that disturb feelings, thoughts, and behavior are called mental disorders. They influence how we interact with the people around us or how we react to stress. So, just like we care for our bodies, we need to pay attention to our mental health as well.

Mental health refers to a person's emotional and social well-being. In simple terms, it means having a healthy brain. Mental health depends on many factors, which we call risk factors. Some risk factors, such as our DNA, are inherited from our parents, while others come from our environments. Environmental factors include life-threatening situations, such as a car accident or experiencing a natural disaster. Our environment is also defined by the people in it. For example, peers might bully us or encourage us to drink alcohol, smoke cigarettes, or consume cannabis.

We all face difficulties throughout our lives, but not all of us develop mental disorders. Mental disorders develop only when too many risk factors accumulate and exceed a threshold level. For example, too much stress, especially at a very young age, can have a negative impact on mental health (Figure 1). In fact, according to the World Health Organization, half of the patients diagnosed with a mental disorder had their first symptoms during adolescence. This shows us that adolescence is a key period for mental health.

\section{WHY IS ADOLESCENCE A KEY PERIOD FOR MENTAL HEALTH?}

Did you know that the brain continues to mature until we are about 25 years old? Recently, scientists found that the brain changes in its structure throughout the adolescent years [1]. The changes in brain structure include forming and removing connections between the brain cells called neurons. These connections allow neurons to communicate with each other and pass along information. The brain eliminates connections that are weak or not in use. This process is called pruning and it is particularly important in adolescence. Pruning helps to strengthen the remaining connections between neurons and increases their efficiency. In this way, the brain selects and preserves information that will be useful for the adult brain. Such changes in connections increase the ability to reason, make decisions, and solve complex problems (Figure 2). 
Figure 1

DNA and the environment affect your mental health. The combination of DNA, which you inherit from your parents, and your experiences impact your mental health. Good experiences, such as being in a caring environment or having supportive relationships, can help your brain to stay healthy. Negative experiences, such as being bullied or consuming cannabis, are risk factors that, in combination with DNA, can influence the brain If too many risk factors exist, you might be pushed beyond a critical level and develop a mental disorder, such as psychosis.

\section{SENSITIVE PERIOD}

A time period during which the brain changes and matures. During this period, the brain is more sensitive to experiences and is changed by them.

\section{PSYCHOSIS}

A mental disorder in which the affected people cannot recognize what is real and what is not.

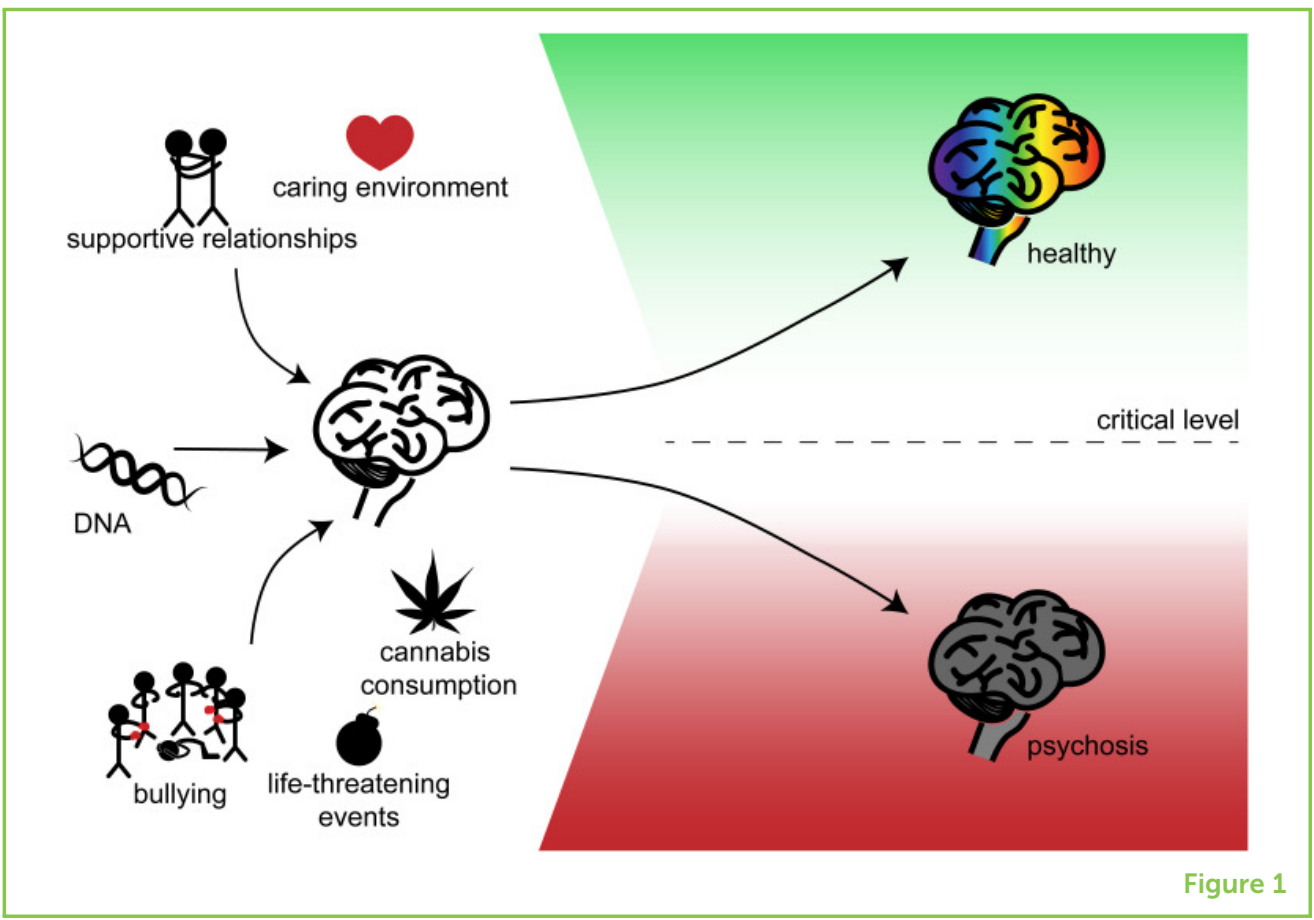

The continuous changes in the adolescent brain are the reason why the adolescent years are considered a sensitive period by scientists. Sensitive periods are specific time windows during development, in which our brains have an increased ability to change in response to life experiences. The brain is more adaptive during our adolescent years, but the changes that occur in an adolescent brain are two-sided. On the one hand, having positive experiences and leading a healthy life can benefit our mental well-being. On the other hand, experiencing unpleasant events can negatively impact our mental health.

One of the mental disorders that may arise during adolescence is called psychosis. About 3 out of 100 people are diagnosed with psychosis in their lifetime [2]. Different risk factors, like your DNA, harmful life events, or the consumption of drugs, such as cannabis, increase the risk of developing psychosis. But what is psychosis? And why do some researchers think that consuming cannabis during adolescence may increase the risk of developing psychosis?

\section{CANNABIS AND PSYCHOSIS: WHAT IS THE CONNECTION?}

Psychosis is a condition in which the affected people lose their grip on reality. They see or hear things that are not real and feel detached from the people around them. They have disorganized thoughts or false beliefs, like being followed by aliens. Psychosis can be triggered by stress, brain injury, infection, or drugs. 
Figure 2

The pruning process in the brain. During development, your body changes and so does your brain. Pruning is an important part of the brain's development and happens from the time you are born until adulthood, but is especially active during adolescence. The three panels show how pruning takes place for one pair of neurons in the brain. During pruning, connections between neurons that are weak or not used (dashed lines) are removed. Pruning is fundamental for brain functions, such as reasoning, making decisions, and solving complex problems.

\section{PSYCHOACTIVE}

\section{SUBSTANCE}

A substance that is active on the brain, changing the brain's function and altering behavior, perception, or mood.

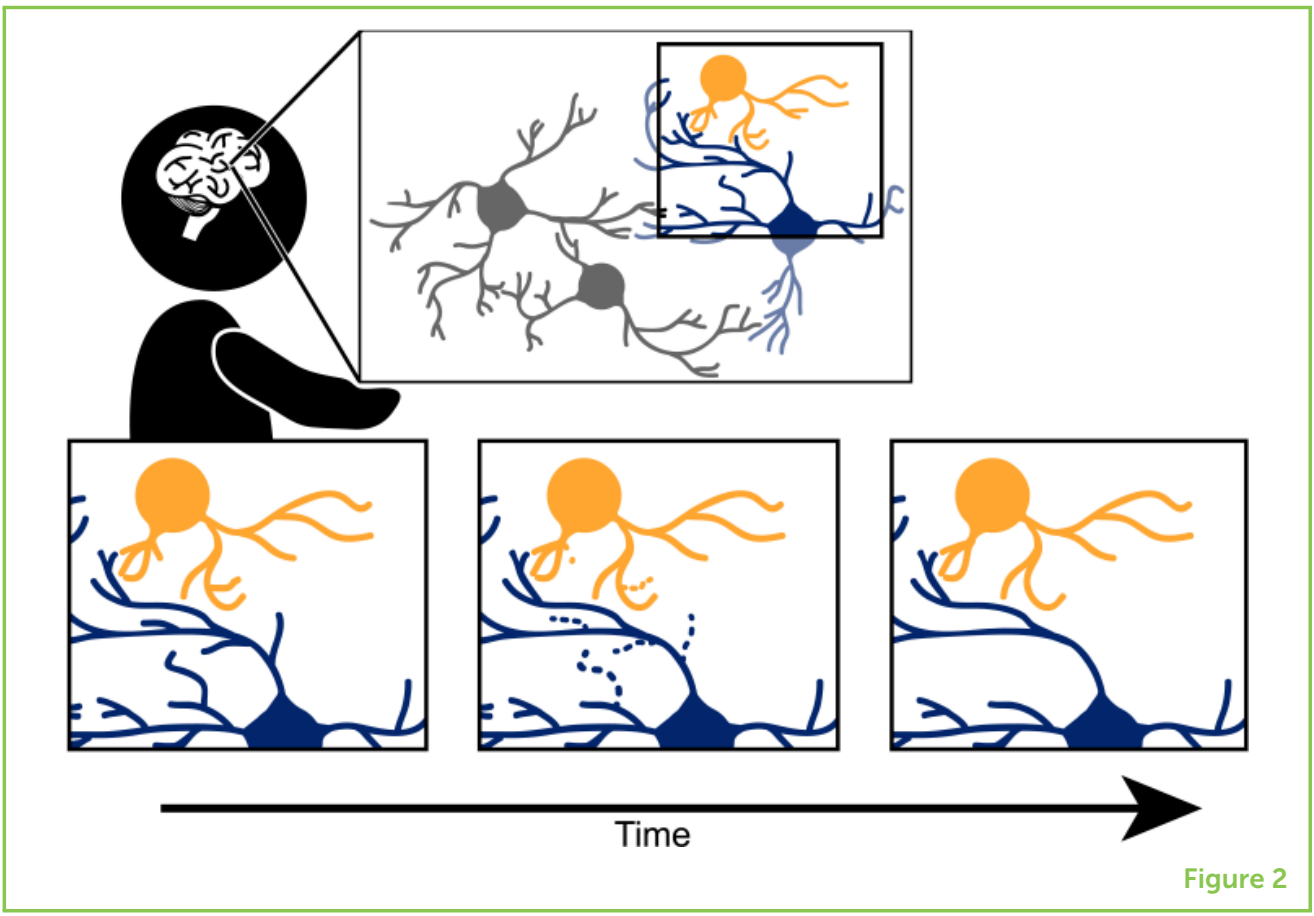

We will focus on one risk factor: drugs, especially cannabis. Cannabis is a psychoactive substance extracted from a plant called Cannabis sativa. Psychoactive substance are drugs that have effects on the brain, changing its function and altering behavior, perception, or mood. In fact, if you talk to someone with psychosis, the chance that that person consumed cannabis during adolescence is higher than in the general population [3].

There are two major theories regarding how cannabis use and psychosis fit together. The first theory is that psychosis happens before cannabis use, and people suffering from psychosis tend to use cannabis as a form of self-medication, to reduce their symptoms. A second theory is that cannabis consumption happens first and leads to psychotic symptoms. Researchers have looked into whether cannabis can lead to psychosis-like symptoms. In one study, they tested people who had previously consumed cannabis but were mentally healthy. One half of the group received the chemical components of cannabis, while the other half received a "fake" drug with no effects. Participants in the cannabis group temporarily had altered perceptions and felt separated from the test environment. This showed a direct effect of cannabis in increasing psychotic symptoms [4]. Studies like these pointed scientists toward the second theory, that cannabis use might lead to psychosis.

However, cannabis use alone cannot explain the onset of psychosis, since not all people who consume cannabis suffer from psychosis. Also, not everyone with psychosis has consumed cannabis. Cannabis use may only add to existing risk factors, such as changes in the DNA that we inherit from our parents (Figure 1). 
Figure 3

The messenger systems and cannabis. (A)

Neurons communicate with each other using specific messenger systems. These are made of ligands (blue and red keys) and receptors (green and yellow locks). Each system has its own specific keys and locks and most of them go from neuron 1 to neuron 2 (A1). Once a neuron is activated by a messenger system, it releases

endocannabinoids, which then travel in the opposite direction, back to neuron 1, and help to tune down the other systems when necessary, using the eCB receptor (A2). This helps to tune down a reaction that is not needed anymore. (B) When cannabis is consumed, THC (black keys) is introduced into the brain. THC activates the eCB receptors even when

endocannabinoids are not around. This shuts down the messenger system in an uncontrolled way, leading to a change in function of the brain.

\section{LIGANDS AND} RECEPTORS

Neurons talk to each other using small chemical substances. Some of them act like tiny keys (scientists call them "ligands"), which fit specialized locks (scientists call them "receptors"). If a ligand binds to a receptor, it may unlock (activated) or lock (inhibit) this neuron.
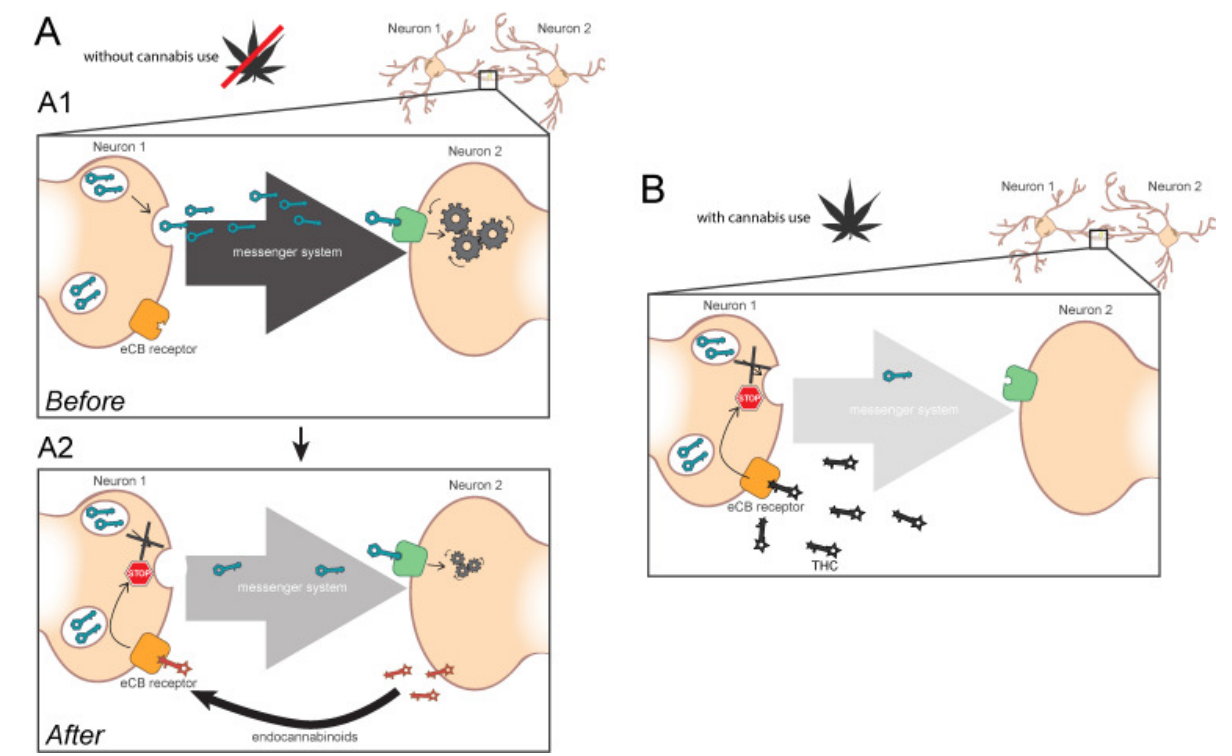

Figure 3

So how does cannabis affect the brain? To find out, let us take a step back and look at some biological mechanisms of communication between neurons in the brain.

\section{HOW DOES CANNABIS AFFECT THE ADOLESCENT BRAIN?}

Brain functions, like perceptions, thoughts, and feelings, are the result of communication between neurons. To efficiently communicate and process information, neurons form connections with each other. Through these connections, they exchange information via messenger molecules. There are many messenger systems in the brain. They consist of two major players: a set of molecules that act as keys (called ligands) and another set of molecules that act as locks (called receptors). Each receptor has a specific ligand like each lock has a specific key. All messenger systems also have molecules called enzymes to break down excess ligands to keep the messengers always in the right amount. Most of the messenger systems communicate in one direction, where the ligand is released from neuron 1 and binds to receptors on neuron 2. There are also some that communicate in the opposite direction, from neuron 2 back to neuron 1. One of the messenger systems that works in the opposite direction is the endocannabinoid (eCB) system. Its ligands are called endocannabinoids and, like all messenger systems, it also contains enzymes to break down excess endocannabinoids. The main function of the eCB system is to maintain a balance in the body by regulating the release of ligands from neuron 1 . When there is a high release of ligands, the eCB system reduces this ligand release, bringing the systems back to a normal state (Figure 3A). 
eCB SYSTEM

A specific messenger system in the brain that keeps all the other messenger systems balanced

THC

One of the molecules of cannabis.
Imagine you are on a rollercoaster ride. Different messenger systems are activated during the ride that make you feel excited or scared. These activations also put the eCB system into action and return all systems to normal levels, thus calming you down after the ride.

The eCB system also plays a crucial role in the development of the brain [5]. We have already learned that adolescence is a period when the brain matures. The eCB system plays a role in this maturation by assisting the process of pruning. Cannabis use during adolescence interacts with the eCB system and thus can alter brain maturation and function.

How exactly does cannabis interfere with brain maturation? Cannabis contains at least 140 different molecules. One of them is delta-9tetrahydrocannabinol, or THC. THC resembles the cannabinoids of the eCB system present in the brain and it binds to the eCB receptors. Think of it as two keys fitting in the same lock. When you consume cannabis, THC levels are high in the brain. The high levels of THC keep the eCB receptors active even when they do not need to be active. While the body's own endocannabinoids are quickly removed when they are not needed, THC is removed very slowly. Hence, continuous use of cannabis leads to a buildup of THC in the brain. Since there are only a fixed number of receptors, the high levels of THC molecules occupy the majority of them (Figure 3B). When this happens, the eCB system is not able to perform its function of maintaining balance. In short, the action of $\mathrm{eCB}$ and $\mathrm{THC}$ is very similar. However, while the proper amount of eCB is produced by your body only when needed, THC comes in uncontrolled amounts and acts even when your body does not need eCB. When cannabis is consumed, many neurons are affected. As a result, the whole brain changes its functionality and the behavior of that person changes. The change in brain functionality can potentially lead to disorders, such as psychosis.

If cannabis has such harmful effects, why does not everyone who consumes cannabis get psychosis? DNA influences how the eCB system responds to cannabis exposure, making people more or less vulnerable to psychosis. Scientists are constantly working to understand how the eCB system works and how it interacts with DNA or harmful life events. These interactions define a person's risk of developing psychosis.

\section{CONCLUSION: TAKE CARE OF YOUR BRAIN!}

The adolescent brain is like a new factory where neurons are still trying to improve their way of communicating. A challenge here and there is always welcome, but too many harmful experiences confuse the brain's messenger systems and may lead to long-lasting communication problems. This confusion paves the way for mental disorders, like psychosis. As cannabis use is a choice we make, it 
is a risk factor that can be easily avoided. The upside is that good experiences during adolescence have a positive influence on later brain functioning. Adolescence is a period when we can set the stage for later emotional and social well-being. So, just like we put on a scarf to avoid getting a cold, we can take care of our mental health as well.

\section{AUTHOR CONTRIBUTIONS}

SL and DP wrote the manuscript. EB prepared the figures. SL, DP, EB, $A C, S H, J K, A K, F S, E H$, and $C W$ contributed to the manuscript revision, read, and approved the submitted version. All authors contributed to the conception and design of the manuscript.

\section{REFERENCES}

1. Fuhrmann, D., Knoll, L. J., and Blakemore, S.-J. 2015. Adolescence as a sensitive period of brain development. Trends Cogn. Sci. 19:558-66. doi: 10.1016/j.tics.2015.07.008

2. Perälä, J., Suvisaari, J., Saarni, S. I., Kuoppasalmi, K., Isometsä, E., Pirkola, S., et al. 2007. Lifetime prevalence of psychotic and bipolar I disorders in a general population. Arch. Gen. Psychiatry 64:19. doi: 10.1001/archpsyc.64.1.19

3. Arseneault, L. 2002. Cannabis use in adolescence and risk for adult psychosis: longitudinal prospective study. BMJ 325:1212-3. doi: $10.1136 / \mathrm{bmj} .325 .7374 .1212$

4. D'Souza, D. C., Perry, E., MacDougall, L., Ammerman, Y., Cooper, T., Wu, Y., et al. 2004. The psychotomimetic effects of intravenous delta-9-tetrahydrocannabinol in healthy individuals: implications for psychosis. Neuropsychopharmacology 29:1558-72. doi: 10.1038/sj.npp.1300496

5. Meyer, H. C., Lee, F. S., and Gee, D. G. 2018. The role of the endocannabinoid system and genetic variation in adolescent brain development. Neuropsychopharmacology 43:21-33. doi: 10.1038/npp.2017.143

SUBMITTED: 28 August 2019; ACCEPTED: 16 March 2020;

PUBLISHED ONLINE: 19 May 2020.

EDITED BY: Daniel W. Wesson, University of Florida, United States

CITATION: Loganathan S, Pöhlchen D, Brivio E, Comes AL, Haas SS, Kalman JL, Krontira AC, Stamp F, Hoch E and Wotjak CT (2020) Be Careful What You Feed Your Brain: Cannabis and Mental Health. Front. Young Minds 8:41. doi: 10.3389/frym. 2020.00041

CONFLICT OF INTEREST: The authors declare that the research was conducted in the absence of any commercial or financial relationships that could be construed as a potential conflict of interest.

COPYRIGHT @ 2020 Loganathan, Pöhlchen, Brivio, Comes, Haas, Kalman, Krontira, Stamp, Hoch and Wotjak. This is an open-access article distributed under the terms 

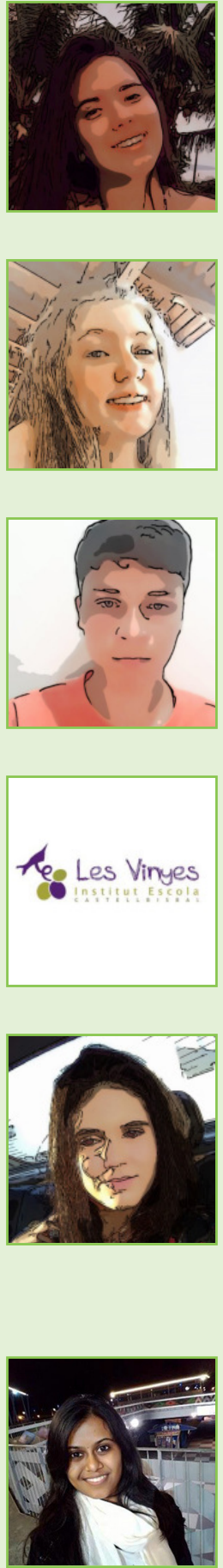

of the Creative Commons Attribution License (CC BY). The use, distribution or reproduction in other forums is permitted, provided the original author(s) and the copyright owner(s) are credited and that the original publication in this journal is cited, in accordance with accepted academic practice. No use, distribution or reproduction is permitted which does not comply with these terms.

\section{YOUNG REVIEWERS}

\section{ANA CLARA, AGE: 14}

I live in Monte Alto city. It is a small town in Brazil and it is famous for its dinosaur museum. I love swimming, watch movies, and TV series. My favorite subjects at Santo Andre High School are English, History, and Biology.

\section{ANA LUISA, AGE: 15}

$\mathrm{Hi}$, my favorite subjects at school are English, History, Chemistry, Physics, and Math. My favorite hobbies are watching movies, TV series, and listening music. I also enjoy hanging out with my friends and I love to travel.

\section{HENRIQUE, AGE: 15}

I study at Santo André High School. During my free time I enjoy playing computer games and stay at my grandparents house.

\section{INSTITUT-ESCOLA LES VINYES, AGE: 15}

We are a fourth ESO classroom formed by 18 students of 14 years old, from the high school "Institut-Escola Les Vinyes" Castellbisbal, Barcelona, Spain. We spend Wednesday afternoons having fun while learning Biology, as our teacher is always trying hard to make it interesting for us.

\section{VITÓRIA, AGE: 14}

I am from Jaboticabal city, Brazil. I study at Santo André High School and I want to become a veterinarian. I love watching TV, hang out with my friends, and spend some of my free time taking care of animals.

\section{AUTHORS}

\section{SRIVAISHNAVI LOGANATHAN}

I am a Ph.D. student at the Max Planck Institute of psychiatry. My work mainly focuses on how changes in calcium channels in the brain can cause mental disorders. Specifically, I am interested in studying mechanisms of calcium channel signaling in different cell types in the brain and their relation to mental disorders. When I am not in the lab, I read novels, write science articles for science outreach, to make science accessible to everyone, and I practice Bharatanatyam, an Indian classical dance. *srivaishnavi@psych.mpg.de 

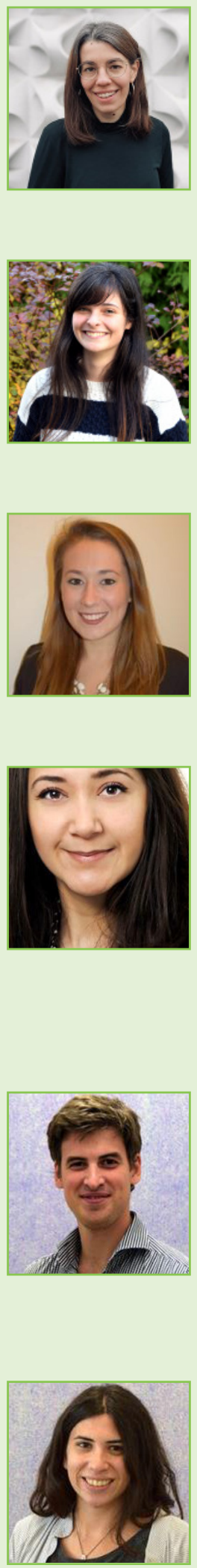

\section{DOROTHEE PÖHLCHEN}

I am a Ph.D. student at the Max Planck Institute for Psychiatry where I work for a large study that aims to identify biological markers of mental disorders. I am specifically interested in how people learn about danger and safety and how this is related to anxiety disorders. When I am not doing research, I like to hike in the Alps and I sing in a choir. *dorothee_poehlchenapsych.mpg.de

\section{ELENA BRIVIO}

I am Ph.D. student working on how the brain processes and responds to stress. I try to understand how different the brains of men and women are in responding to stress and how these processes might lead to the development of mental disorders. My favorite way of relaxing in my free time is baking cookies and cakes, but I also love to bring science to the public.

\section{ASHLEY L. COMES}

I am a Ph.D. student working in the field of psychiatric genomics. My work revolves around understanding the involvement of environmental exposures, like stressful life events, and epigenetic mechanisms on the course of severe mental illness. In my free time, I enjoy taking long walks with my fluffy pup Roo and unleashing my creative side through painting.

\section{SHALAILA S. HAA}

I have recently finished my Ph.D. at the Psychiatry Clinic of the Ludwig-Maximilians-University in Munich. Here, I investigated the effects of a computerized social cognitive training (SCT) paradigm on cognition, symptoms, and neural mechanisms in patients with early psychosis. Using multivariate pattern analysis, I also developed a model that could monitor response to SCT by stratifying individuals likely to profit from this training paradigm based on changes in their resting-state functional connectivity pattern.

\section{JANOS L. KALMAN}

I am a fourth year psychiatry resident and a Ph.D. student. My research work revolves around gaining a better understanding of the genetic factors influencing the disease course in psychotic disorders. For example, we are currently investigating the genetic determinants of age at disease onset in bipolar disorder. I am also interested in how digitalization (e.g., of medical notes) can be used to assist and improve patient care and research.

\section{ANTHI C. KRONTIRA}

I am a Ph.D. student at the Max Planck Institute of Psychiatry working on the link between prenatal stress and the risk for the development of psychiatric disorders later in life. Specifically, I study the molecular mechanisms through which maternal stress and/or psychopathology during pregnancy affect brain development of the embryo, using three-dimensional human brain organoids. When I am not trying to understand the brain, I swim and explore the underwater world. 

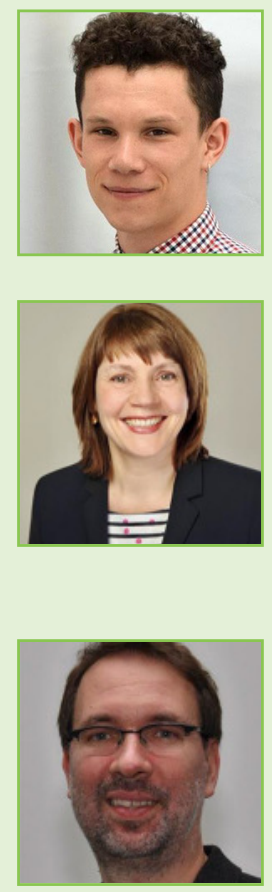

\section{FABIAN STAMP}

I am a Ph.D. student at the Max Planck Institute of Psychiatry. In my research I investigate mechanisms that enable the brain cells to rapidly adapt to changes and that are important for brain development, learning, and memory. Besides my research, I am interested in making science accessible for people of all ages.

\section{EVA HOCH}

I am a researcher in the field of cannabis and cannabinoids and the head of the Cannabinoid Treatment and Research Group and lead psychologist at the Department of Psychiatry, Ludwig-Maximilian University in Munich, Germany. I conducted large studies on cannabis, alcohol, and nicotine dependence. My recent work also addresses the medicinal benefits of cannabinoids.

\section{CARSTEN T. WOTJAK}

I was born and grew up in Leipzig (East Germany) and La Habana (Cuba). Since my study of Biology, I am fascinated by neurobiological underpinnings of behavior. I had the luck and pleasure to follow this up during my Ph.D. and post-doctoral studies in Munich, Hamburg, and London. My research group at the Max-Planck Institute of Psychiatry works on animal models of psychiatric diseases. We hope that our work on the fear-buffering role of the endogenous cannabinoid system will find its way into the clinical practice.

${ }^{\dagger}$ These authors have contributed equally to this work

‡Present address: Carsten T. Wotjak, Boehringer Ingelheim Pharma GmbH \& Co KG, Biberach, Germany 